Abstract 008 Table 1

\begin{tabular}{llllc}
\hline & $\begin{array}{l}\text { HFNEF } \\
\text { with LVH }\end{array}$ & $\begin{array}{l}\text { HFNEF without } \\
\text { LVH }\end{array}$ & Controls & $\begin{array}{l}\text { p-value } \\
\text { (ANOVA) }\end{array}$ \\
\hline $\begin{array}{l}\text { SD systolic motions } \\
\text { (ms) at Rest }\end{array}$ & $53.3 \pm 32.7$ & $45.5 \pm 33.2$ & $44.8 \pm 25.7$ & 0.456 \\
$\begin{array}{l}\text { SD systolic motions } \\
\text { (ms) on exercise }\end{array}$ & $48.0 \pm 28.3^{*}+$ & $28.7 \pm 18.7$ & $25.7 \pm 15.7$ & $<0.001$ \\
\hline
\end{tabular}

${ }^{*} \mathrm{p}<0.05$ compared to controls.

$t p<0.05$ compared to HFNEF patients without LVH.

\section{UNCOVERING THE MECHANISM OF THE PARADOXICAL ASSOCIATION BETWEEN CARDIAC DYSSYNCHRONY AND BETTER SURVIVAL IN HEART FAILURE}

doi:10.1136/heartjnl-2012-301877b.9

R J Jabbour, ${ }^{*}$ J Shah, J Mayet, D P Francis. Imperial College Healthcare Trust, UK

Introduction Paradoxically, dyssynchrony before CRT is associated with a better prognosis. We tested whether this was dependent on device implantation or on how the cohort was defined ( $E F \leq 35$ vs All-comers).

Methods 419 patients (67.8 \pm 11.3 years, $79.2 \%$ males, 127 deaths) with heart failure had echocardiographic assessment of mechanical dyssynchrony and were followed up (median 3.1 years).

Results 135 had dyssynchrony and 62 received CRT. The mean EF was $33.1 \pm 15.0 \%$; 157 (35.2\%) had an $\mathrm{EF}>35 \%$. Among patients with $E F \leq 35 \%(n=249)$, shorter aortic pre-ejection time (ie, less dyssynchrony) was associated with a worse prognosis $(p<0.05)$. All dyssynchrony markers were higher in survivors $(p<0.001$ by sign test, upper panel). EF was not prognostic and depressed by dyssynchrony $(\mathrm{r}=-0.4, \mathrm{p}<0.001)$. By examining all patients (regardless of EF); the association between dyssynchrony and better survival disappeared ( $p>0.05$, lower panel). EF was restored to its prognostic significance $(p=0.02)$. Taking a different approach to define poor ventricular function-using low S-wave velocity-EF had prognostic significance $(p<0.05)$ and dyssynchrony markers were non-prognostic ( $p>0.05)$.

Conclusion Dyssynchrony predicts better survival in low EF groups because dyssynchrony artifactually lowers EF without damaging

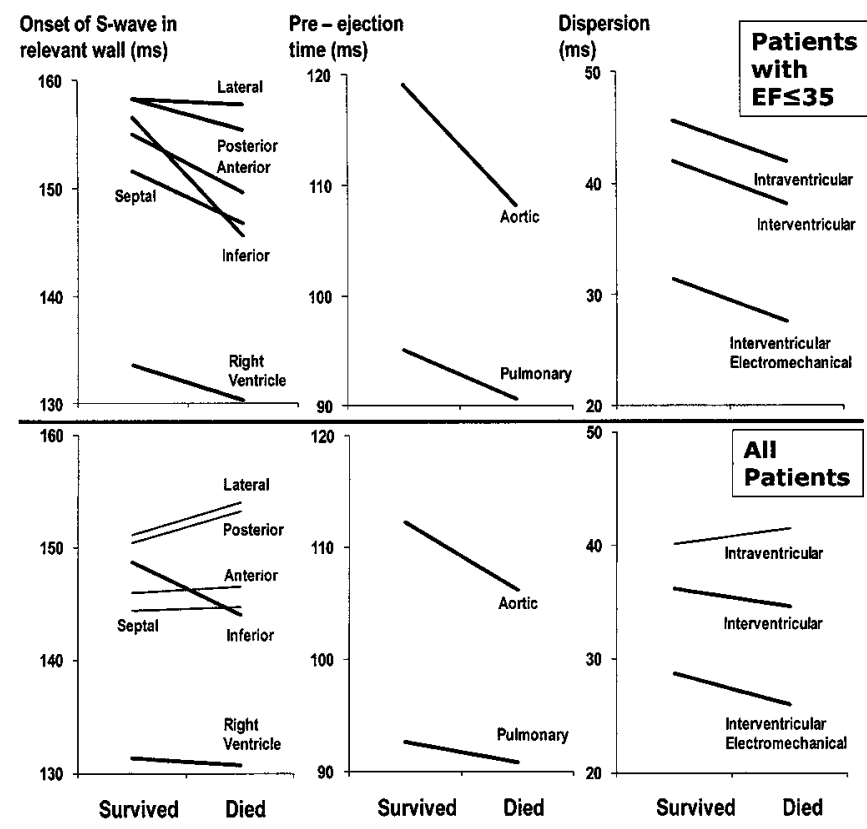

Abstract 009 Figure 1 survival. The effect is independent of CRT. Replacement of EF with dyssynchrony-neutral measures of LV function, for example, peak Swave velocity would avoid the appearance that dyssynchrony is favourable.

\section{MULTICENTRE VALIDATION OF THE ADVERSE PROGNOSTIC IMPLICATIONS OF DECLINING SERUM ALBUMIN LEVELS IN CHRONIC HEART FAILURE}

doi:10.1136/heartjnl-2012-301877b.10

${ }^{1} \mathrm{R} \mathrm{J}$ Jabbour, ${ }^{2} \mathrm{~S}$ Husain, ${ }^{1} \mathrm{~N}$ Zaman, ${ }^{1} \mathrm{~N}$ Aung, ${ }^{2} \mathrm{H} \mathrm{Z}$ Ling, ${ }^{1} \mathrm{R}$ Baruah, ${ }^{1} \mathrm{G}$ Cole, ${ }^{1} \mathrm{C}$ Manisty, ${ }^{1} \mathrm{~A}$ Barron, ${ }^{1} \mathrm{~J}$ Mayet, ${ }^{1} \mathrm{D}$ Francis, ${ }^{1}$ Martin Thomas, ${ }^{1} \mathrm{~S}$ Woldman, ${ }^{1} \mathrm{D} 0$ Okonko. ${ }^{1}$ Imperial College Healthcare Trust, UK; ${ }^{2}$ University College London Hospital, London, UK

Background Single-centre studies have shown that a low serum albumin at baseline forecasts enhanced mortality in chronic heart failure (CHF) possibly because it reflects aberrations (eg, inflammation, impaired nutrition, plasma volume expansion) that can exacerbate disease. We hypothesised that attenuations in serum albumin over time would be prognostically more ominous than baseline values, and would be so even in a multicentre setting.

Methods We analysed the survival implications of baseline albumin and $\Delta$ albumin in a derivation cohort of 246 CHF outpatients (mean [ $\pm \mathrm{SD}]$ age $68 \pm 12$ years, LVEF $29 \pm 8 \%, 48 \%$ NYHA class $>2$ ) from University College London Hospital and then in a validation cohort of $148 \mathrm{CHF}$ outpatients (age 69 \pm 12 years, LVEF $28 \pm 10 \%, 41 \%$ NYHA class $>2$ ) from Imperial Healthcare (St Marys Hospital and Hammersmith Hospital, London).

Results In the derivation cohort, 51 (21\%) patients died over 13 months. Baseline albumin independently predicted mortality (HR 0.89, 95\% CI 0.84 to $0.94, \chi^{2}: 18, \mathrm{p}<0.0001$ ). However, $\Delta$ albumin (unadjusted HR $0.89,95 \%$ CI 0.84 to $0.92, \chi^{2}: 53$, $\mathrm{p}<0.0001$ ) was even more predictive (Difference in ROC AUC for baseline vs $\Delta$ albumin $0.16, p<0.001$ ) and did so independently of all covariates including baseline albumin. A reduction in albumin $>6 \mathrm{~g} / 1$ optimally predicted death (ROC AUC 0.82, $\mathrm{p}<0.0001$ ) and conferred a sixfold escalated risk of mortality (HR 6.42, 95\% CI 3.67 to $11.22, \mathrm{p}<0.0001)$. In incremental prognostic analyses, the addition of $\Delta$ albumin to the strongest four variable model (baseline albumin, NYHA class, $\Delta$ urea, $\Delta$ haemoglobin) dramatically augmented the $\chi^{2}$ value (43 vs $84, p<0.0001$ ). In the validation cohort, $43(30 \%)$ patients died. $\Delta$ albumin (unadjusted HR $0.89,95 \%$ CI 0.86 to $0.92, \chi^{2}: 44, p<0.0001$ ) was again prognostically superior to baseline albumin with a fall $>6 \mathrm{~g} / \mathrm{l}$ predicting an $\sim$ sixfold increased risk (HR 5.64, 95\% CI 3.08 to 10.31, $\chi^{2}: 35, \mathrm{p}<0.0001$ ). Addition of $\Delta$ albumin to the strongest three variable model (baseline red cell distribution width, $\Delta$ red cell distribution width, $\Delta$ urea) also augmented the $\chi^{2}$ value ( 51 vs $65, \mathrm{p}<0.001$ ).

Conclusions A fall in serum albumin over time consistently predicts an amplified risk of death in systolic CHF and enables simple and cheap risk stratification.

\section{HBA1C AND MORTALITY IN DIABETIC INDIVIDUALS WITH HEART FAILURE: AN OBSERVATIONAL COHORT STUDY}

doi:10.1136/heartjnl-2012-301877b.11

D H J Elder, ${ }^{*}$ L Donnelly, A Wong, B R Szwejkowski, M Pauriah, T K Lim, S D Pringle, A Choy, E Pearson, A Morris, J George, A Struthers, C Palmer, A Doney, C C Lang. University of Dundee, Dundee, UK

Background Controversy exists regarding the importance of glycaemic control in patients with type 2 diabetes mellitus (T2DM) and chronic heart failure (CHF) based on conflicting reports that had used a single baseline HbA1c. 